\title{
Error Self-Calibration Algorithm for Acoustic Vector Sensor Array
}

\author{
Peng Wang $\mathbb{D}$, Yujun Kong, and Mingxing Zhang \\ Department of Mathematics, North University of China, Taiyuan, Shanxi 030051, China \\ Correspondence should be addressed to Peng Wang; wpmath@nuc.edu.cn
}

Received 25 January 2019; Accepted 29 July 2019; Published 4 September 2019

Academic Editor: Paolo Bruschi

Copyright (c) 2019 Peng Wang et al. This is an open access article distributed under the Creative Commons Attribution License, which permits unrestricted use, distribution, and reproduction in any medium, provided the original work is properly cited.

In this paper, the errors of acoustic vector sensor array are classified, the impact factor of each error for the array signal model is derived, and the influence of each type of error on the direction-of-arrival (DOA) estimation performance of the array is compared by Monte Carlo experiments. Converting the directional error and location error to amplitude and phase errors, the optimization model and error self-calibration algorithm for acoustic vector sensor array are proposed. The simulation experiments and field experiment data processing of MEMS vector sensor array show that the proposed self-calibration algorithm has good parameter estimation performance and certain engineering practicability.

\section{Introduction}

Compared to traditional acoustic pressure sensor, the acoustic vector sensor can measure both the scalar acoustic pressure and the acoustic particle velocity vector at a certain point of the acoustic field. So, it possesses higher direction sensitivity and can acquire more measurement information $[1,2]$.

A novel biomimetic MEMS vector hydrophone in Figure 1 has been developed for acoustic detection application [3-6], which is based on the meso-piezoresistive effect and the acoustics theory of cylinder. It exploits the thin film of quantum as well as the sensitive unit, which adopts cilia bionic structure. The bionic structure is adapted by the combination of micro and nano cilia, and the underwater acoustic signal is detected by detecting the change of resistance of the four-beam microstructures. Through the experimental tests, the sensitivity of MEMS vector hydrophone can reach up to $-191.9 \mathrm{~dB}$ and $-188.1 \mathrm{~dB}(0 \mathrm{~dB}=1 \mathrm{~V} / \mu \mathrm{Pa})$, which conforms to the sensitivity increments of $6 \mathrm{~dB}$ per octave in the working bandwidth ranging from $20 \mathrm{~Hz}$ to $1 \mathrm{KHz}$, and the directivity curve follows " 8 " cosine orientability, which conforms to avoid the left-right ambiguity. Because the micronano-sensitive structure can realize batch manufacturing and one-time integration, the MEMS vector hydrophone has the characteristics of small size, good consistency, and better suitability for the construction of arrays.
By taking advantage of the extra information, acoustic vector sensor array is able to improve the direction-ofarrival (DOA) estimation performance without increasing array aperture size. Nehorai and Paldi have developed the measurement model of the acoustic vector sensor array for dealing with narrowband sources [1]; many methods such as MUSIC algorithm and root-MUSIC algorithm have been proposed for applying acoustic vector sensor array to DOA estimation problems [6-9].

In most traditional DOA estimation algorithms, the array steering matrix is all known, so these algorithms have excellent performance. However, in practice, due to various factors, all types of error are inevitable, so the array steering matrix will always be a certain degree of deviation or disturbance. Therefore, the performance of these high resolution DOA estimation algorithms will be seriously deteriorated and will even fail. In [6], a self-adapting root-MUSIC algorithm is proposed and successfully applied in lake trials of MEMS vector hydrophone array. In order to ensure the estimation accuracy of the proposed algorithm, we corrected the array which is composed of two MEMS hydrophones in advance, so we got better results. But if the array error is not corrected, the performance of the algorithm will be greatly reduced.

So, array error is a technical bottleneck when these methods are be applied in practical. In the early days, array error correction was realized by discrete measurement, 


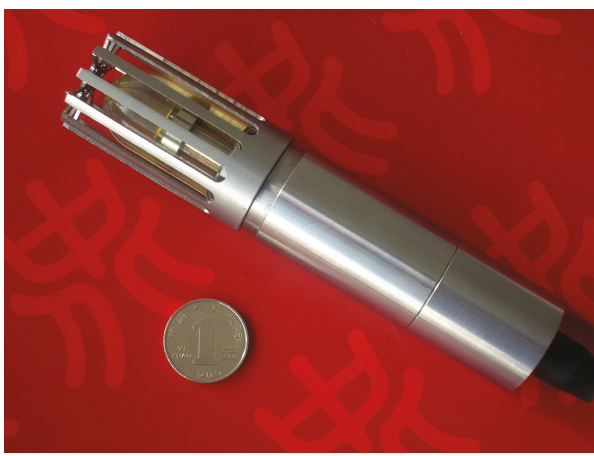

(a) Vector sensor

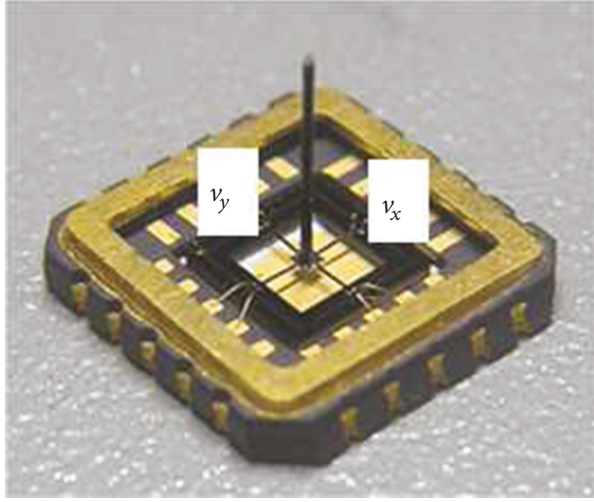

(b) Microstructure

FIGURE 1: MEMS vector sensor and microstructure.

interpolation, and storage of array steering matrix, but these methods are costly and ineffective. Since 1990s, some scholars convert array error correction to a parameter estimation problem through the modeling of array disturbances. These array error calibration methods can be divided into two categories: auxiliary source correction [10] and adaptive correction [11]. Auxiliary source correction (ASC) estimates the array disturbance parameters offline by setting an auxiliary source in space. However, ASC method requires very high accurate azimuth information of the auxiliary source. When the azimuth information of the auxiliary source is biased, it will bring about large deviation. The adaptive correction method to carry out the joint estimation to the azimuth of the signal source and the array perturbation parameters is realized according to some optimization functions, which do not need the auxiliary source with known azimuth information, and can be corrected online in real time to complete the azimuth estimation, so its correction accuracy is higher, but it also brings a large amount of computation.

Many scholars have done many researches about the array error correction. Liu et.al describe the attitude error of the array elements on the basis of using the Generalized Euler Angle from kinematics and built Gauss Perturbation Models of array errors [12]. Liu and Jin proposed an error correction for phase error in vector sensor array by using the projection of array steering matrix in signal subspace [13]. Liang et.al proposed an array correction method based on iterative optimization and subspace fitting and combined processing by using pressure and particle velocity [14]. Lim et.al presented two algorithms that are capable of estimating and compensating rotational perturbation angles based on steering vector error subspace criteria [15]. Liang et al. presented a self-calibration algorithm of altitude error, which can estimate the attitude error parameter and DOA of sources simultaneously by utilizing nonlinear iteration to minimize the objective function [16]. Zhang et al. proposed a calibration algorithm based on eigendecomposition for location, gain, and phase errors of acoustic vector sensor array, which require at least three DOA information from the cooperation source [17].
But these methods are according to the acoustic pressure sensor array that the sensor does not have in directivity. But for the vector sensor array, each sensor has directional errors leading to the array which also has directional errors. The main types can be classified into three categories. The first type is amplitude and phase distortion, which needs to consider the error between the different sensors and the error between the acoustic pressure and the vibration velocity of one sensor. The second type is directional distortion including the directional deviation of the different sensors and directional deviation between vibration velocities $v_{x}$ and $v_{y}$ of one sensor. The third type is the position distortion. This is the position deviation of sensor placement in practical application.

In this paper, the amplitude error, phase error, directional error, and position error are discussed, and a new error self-calibration algorithm for acoustic vector sensor array is proposed. The organization of this paper is as follows: the signal model, MUSIC algorithm, error model of AVS array, and the influences for the performance of DOA estimation are presented and discussed in Section 2; the error self-calibration algorithm is proposed in Section 3; the simulation experiments and lake test of MEMS vector sensor array are made in Section 4; and the paper is summarized in Section 5.

\section{Models}

2.1. Signal Model of AVS Array. Consider $N$ far-field narrowband signals' incident on an uniform line array of M2-D acoustic vector sensor along the $x$-axis in geometric plane surface (Figure 2), from directions $\boldsymbol{\theta}=\left[\theta_{1}, \theta_{2}, \cdots, \theta_{N}\right]^{\mathrm{T}}$, the received signal vector of the array can be expressed as follows:

$$
\mathbf{Z}(t)=\mathbf{A}(\boldsymbol{\theta}) \mathbf{S}(t)+\mathbf{n}(t)
$$

where $\mathbf{Z}(t)$ is the $3 M \times 1$ snapshot data vector of the array, $\mathbf{S}(t)$ is the $N \times 1$ vector of the signal, $\mathbf{n}(t)$ is the 3 $M \times 1$ vector of the Gaussian noise data vector, the noise 


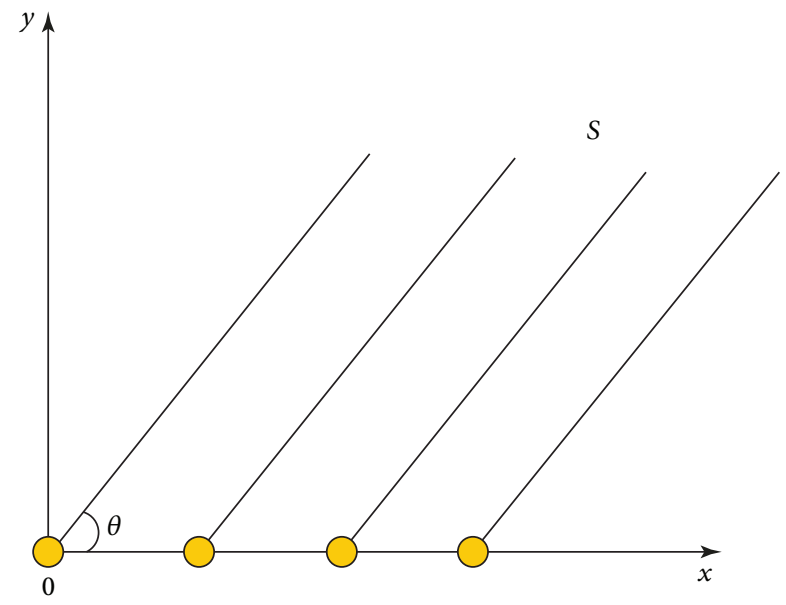

Figure 2: MEMS vector sensor and microstructure.

and the signal are independent, and $\mathbf{A}(\theta)$ is the $3 M \times N$ steering vector matrix of the acoustic vector sensor array

$$
\mathbf{A}(\boldsymbol{\theta})=\left[\mathbf{a}\left(\theta_{1}\right), \mathbf{a}\left(\theta_{2}\right), \cdots, \mathbf{a}\left(\theta_{N}\right)\right],
$$

where $\mathbf{a}\left(\theta_{k}\right)=\left[1, \mathrm{e}^{\mathrm{j} \beta_{k}}, \mathrm{e}^{2 j \beta_{k}}, \cdots, \mathrm{e}^{(M-1) j \beta_{k}}\right]^{\mathrm{T}} \otimes \mathbf{u}_{k}, k=1,2, \cdots, N$, is the acoustic pressure corresponding of $k$ th signal, $\beta_{k}=$ $(2 \pi / \lambda)\left(d \sin \theta_{k}\right)$, in which $d$ is the interelement spacing, and $\lambda$ is the wavelength corresponding to the maximum frequency of signals. $\mathbf{u}_{k}=\left[1, \cos \theta_{k}, \sin \theta_{k}\right]^{\mathrm{T}}$ is the direction vector of the $k$ th signal, and the notation $\otimes$ denotes the Kronecker product.

The covariance matrix of $\mathbf{Z}(\mathbf{t})$ is given by

$\mathbf{R}=\mathrm{E}\left[\mathbf{Z}(t) \mathbf{Z}^{H}(t)\right]=\mathbf{A E}\left[\mathbf{S}(t) \mathbf{S}^{H}(t)\right] \mathbf{A}^{H}+\sigma_{n}^{2} \mathbf{I}=\mathbf{A R}_{s} \mathbf{A}^{H}+\sigma_{n}^{2} \mathbf{I}$,

where $\mathbf{R}_{S}$ is signal covariance matrix, $\sigma^{2}$ is the power of Gaussian white noise, and

$$
\mathbf{I}_{v}=\left[1, \frac{1}{2}, \frac{1}{2}, \cdots, 1, \frac{1}{2}, \frac{1}{2}\right]
$$

is the normalized noise covariance matrix.

By the definition of the array covariance, $\mathbf{R}$ is a Hermitian matrix, so the eigenvalues $\lambda_{i}(i=1,2, \cdots, 3 M)$ are all positive. Suppose $\lambda_{1} \geq \lambda_{2} \geq \cdots \geq \lambda_{3 M}>0$, and the corresponding eigenvector is $\mathbf{e}_{1}, \mathbf{e}_{2}, \cdots, \mathbf{e}_{3 M}$, then the eigendecomposition of $\mathbf{R}$ is

$$
\mathbf{R}=\mathbf{U} \sum \mathbf{U}^{H}=\sum_{m=1}^{3 M} \lambda_{m} \mathbf{e}_{m} \mathbf{e}_{m}^{H}
$$

where $\boldsymbol{\Sigma}=\operatorname{diag}\left(\lambda_{1}, \lambda_{2}, \cdots, \lambda_{3 M}\right)$ is a diagonal matrix composed of all eigenvalues of matrix $\mathbf{R}$ and $\mathbf{U}=\left[\mathbf{e}_{1}, \mathbf{e}_{2}, \cdots, \mathbf{e}_{3 M}\right]$ is a matrix composed of eigenvectors of matrix $\mathbf{R}$.

The eigenvalues of the covariance matrix $\mathbf{R}$ can be used to distinguish the signal and noise, the $3 M$ eigen- values are divided into the front $N$ large eigenvalues $\lambda_{1}$ $\geq \lambda_{2} \geq \cdots \geq \lambda_{N}>\sigma_{n}^{2}$ and $3 M-N$ smaller eigenvalues, in which one part is $\sigma_{n}^{2}$ and the other part is $\sigma_{n}^{2} / 2$, and the corresponding eigenvectors are constituted in the matrix $\mathbf{U}_{s}=\left[\mathbf{e}_{1}, \mathbf{e}_{2}, \cdots, \mathbf{e}_{N}\right]$ and $\mathbf{U}_{n}=\left[\mathbf{e}_{N+1}, \mathbf{e}_{N+2}, \cdots, \mathbf{e}_{3 M}\right]$, respectively, which are called the signal subspace and the noise subspace. In theory, the signal subspace and the noise subspace are orthogonal.

2.2. MUSIC Algorithm for AVS Array. From the theory of subspace decomposition, formula (5) can be written as

$$
\mathbf{R}=\mathbf{U}_{s} \sum_{s} \mathbf{U}_{s}^{H}+\mathbf{U}_{n} \sum_{n} \mathbf{U}_{n}^{H}=\sum_{m=1}^{N} \lambda_{m} \mathbf{e}_{m} \mathbf{e}_{m}^{H}+\sum_{m=N+1}^{3 M} \lambda_{m} \mathbf{e}_{m} \mathbf{e}_{m}^{H} .
$$

It is obvious that each column vector of array steering matrix is located in the signal subspace, so the signal subspace $\mathbf{U}_{s}$ is the same as the space spanned by the column vector of signal subspace array steering matrix $\mathbf{A}(\theta)$, then $\mathbf{A}(\theta)$ and noise subspace $\mathbf{U}_{n}$ are orthogonal,

$$
\mathbf{a}^{H}(\theta) \mathbf{U}_{n}=0 .
$$

In practice, considering the finite sample data, the vector sensor covariance matrix can be estimated as

$$
\tilde{\mathbf{R}}=\frac{1}{L} \sum_{i=1}^{L} \mathbf{Z}(t) \mathbf{Z}^{H}(t)
$$

where $L$ is the number of snapshot. And due to the existence of noise, $\mathbf{a}(\theta)$ and $\mathbf{U}_{n}$ cannot be completely orthogonal; DOA estimation of the MUSIC algorithm is actually achieved by minimum optimization

$$
\tilde{\boldsymbol{\theta}}_{\mathrm{MUSIC}}=\arg \min \mathbf{a}^{H}(\boldsymbol{\theta}) \tilde{\mathbf{U}}_{n} \tilde{\mathbf{U}}_{n}^{H} \mathbf{a}(\boldsymbol{\theta}),
$$

and the spectral estimation formula of MUSIC algorithm is

$$
\tilde{\boldsymbol{\theta}}_{\text {MUSIC }}=\arg \max \frac{1}{\mathbf{a}^{H}(\boldsymbol{\theta}) \tilde{\mathbf{U}}_{n} \tilde{\mathbf{U}}_{n}^{H} \mathbf{a}(\boldsymbol{\theta})} .
$$

\subsection{Error Model and Simulation for AVS Array}

2.3.1. Amplitude Distortion. The array amplitude distortion includes the amplitude distortion between the sensors and the amplitude distortion between the components of the sensors. Ideally, it is consistent between the sensitivity of each sensor and between different components of the sensors. However, due to the processing technology and other issues, it is not be completely consistent. The sensitivity of the $m$ th vector sensor is

$$
\mathbf{g}_{m}=\left[g_{m p}, g_{m v_{x}}, g_{m v_{y}}\right]^{\mathrm{T}}, m=1,2, \cdots, M
$$




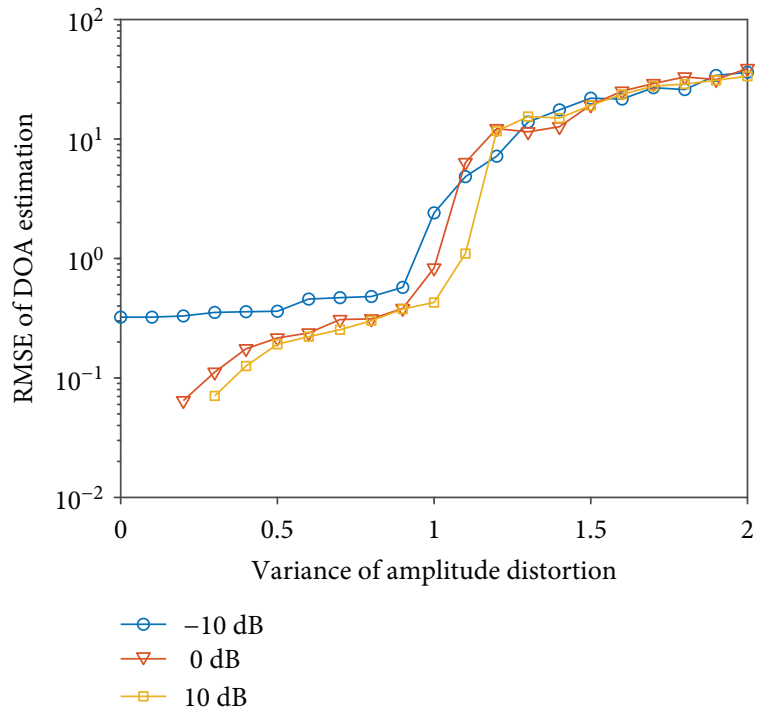

FIGURE 3: The curves between RMSE of DOA estimation and the variance of amplitude distortion with different SNRs.

where $g_{m p}, g_{m v_{x}}$, and $g_{m v_{y}}$ are the sensitivities of the acoustic pressure component $p$, the vibration velocity component $v_{x}$, and the vibration velocity $v_{y}$, respectively, in the $m$ th sensor

$$
\mathbf{G}=\operatorname{diag}\left(g_{1 p}, g_{1 v_{x}}, g_{1 v_{y}}, g_{2 p}, g_{2 v_{x}}, g_{2 v_{y}}, \cdots, g_{M p}, g_{M v_{x}}, g_{M v_{y}}\right) \text {. }
$$

Suppose a single signal from a 40-degree incident on an uniform line array composed of 6 MEMS vector hydrophones, the interelement spacing is half wavelength, the adding noise is Gaussian white noise, SNR being $-10 \mathrm{~dB}, 0 \mathrm{~dB}$, and $10 \mathrm{~dB}$, respectively, and the amplitude distortion satisfies the normal distribution in which the mean value is 0 and the variance is constant. When the variance of amplitude distortion changes from 0 to 2, using 500 independent Monte Carlo trials for each value, the curves between the RMSE of DOA estimation and the variance of amplitude distortion are shown in Figure 3; it can be seen that the RMSE of DOA estimation becomes large with the increase of variance of array amplitude distortion, and the performance is close for different SNRs. This shows that the influence for the performance of DOA estimation from array amplitude distortion is more serious than from the noise.

2.3.2. Phase Distortion. The phase error includes the phase error of the elements and the phase error of the component of elements. Due technological reasons, the phase of the sensor and the different components of the sensor are not the same.

Suppose the phase response of the $m$ th vector sensor is

$$
\mathrm{e}^{j \phi_{m}}=\left[\mathrm{e}^{j \varphi_{m p}}, \mathrm{e}^{j \varphi m v_{x}}, \mathrm{e}^{j \varphi m v_{y}}\right]^{\mathrm{T}},
$$

in which $\mathrm{e}^{j \varphi_{m p}}, \mathrm{e}^{j \varphi m v_{x}}$, and $\mathrm{e}^{j \varphi m v_{y}}$ are the phase responses of the acoustic pressure $p$, the vibration velocity component $v_{x}$,

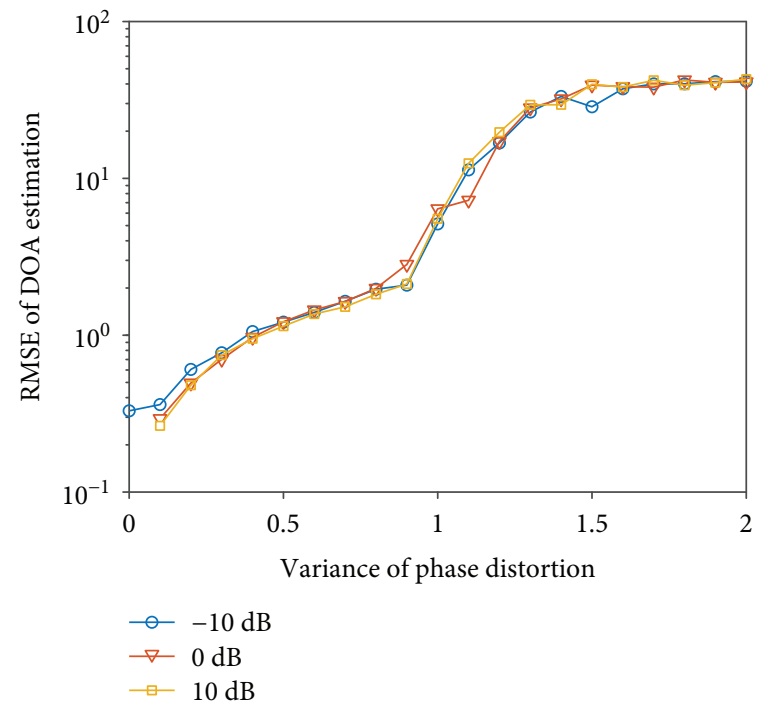

FIgURE 4: The curves between RMSE of DOA estimation and the variance of phase distortion with different SNRs.

and the vibration velocity $v_{y}$, respectively, in the $m$ th sensor, so the phase response of the vector sensor array is

$\boldsymbol{\Phi}=\operatorname{diag}\left(\mathrm{e}^{j \phi 1 p}, \mathrm{e}^{j \phi 1 v_{x}}, \mathrm{e}^{j \phi 1 v_{y}}, \mathrm{e}^{j \phi 2 p}, \mathrm{e}^{j \phi 2 v_{x}}, \mathrm{e}^{j \phi 2 v_{y}}, \cdots, \mathrm{e}^{j \phi_{m p}}, \mathrm{e}^{j \phi_{m v x}}, \mathrm{e}^{j \phi_{m v y}}\right)$.

Under the above simulation conditions, suppose the phase distortion satisfies the norm distribution with the mean value is 0 and the variance is constant. When the variance of phase distortion changes from 0 to 2 , the curves between the RMSE of DOA estimation and the variance of phase distortion are shown in Figure 4 under 500 independent Monte Carlo trials for each value. It can be seen that the performance of DOA estimation under different SNRs is almost the same, which shows that the influence of phase distortion on array performance is more serious than that of noise. Even when the variance of phase error is small, the RMSE of DOA estimation is larger.

2.3.3. Directional Distortion. An acoustic vector sensor has to be of an intrinsic character in the directional sensitivity. Ideally, the output of the single vector sensor is

$$
q=[1, \cos (\theta), \sin (\theta)]^{\mathrm{T}} .
$$

When the array (such as linear array) is composed, the vibration velocity component of each sensor is pointed to the same direction, and the components of the vibration velocity $v_{x}$ and the vibration velocity $v_{y}$ are vertical. But in practice, it cannot completely guarantee the full alignment of the vibration velocity components, so it will bring the distortion of the direction of the sensor. At the same time, for the combined vector sensor, the orthogonality between the components of the vibration velocity and the vibration velocity cannot be guaranteed, so it can cause directional distortion. 


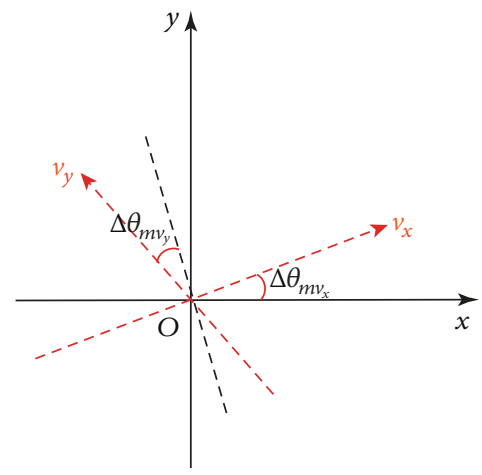

FIgURE 5: The diagram of directional distortion.

Under the influence of the directional distortion, the output of the $m$ th vector sensor is

$\mathbf{q}_{m}=\left[\begin{array}{c}1 \\ q_{m v_{x}} \\ q_{m v_{y}}\end{array}\right]=\left[\begin{array}{c}1 \\ \cos \left(\theta+\Delta \theta_{m x}\right) \\ \sin \left(\theta+\Delta \theta_{m x}+\Delta \theta_{m y}\right)\end{array}\right], m=1,2, \cdots, M$,

in which $\Delta \theta_{m x}$ indicates the direction deviation of the vibration velocity $v_{x}$ and $\Delta \theta_{m y}$ indicates the vertical deviation of the components of the vibration velocity $v_{x}$ and vibration velocity $v_{y}$ in Figure 5.

The distortion matrix of the vector sensor array influenced by the directional error of elements is

$$
\mathbf{Q}=\operatorname{diag}\left(1, q 1 v_{x}, q 1 v_{y}, 1, q 2 v_{x}, q 2 v_{y}, \cdots, 1, q M v_{x}, q M v_{y}\right) .
$$

Similar to the above simulation conditions, the curves between the RMSE of DOA estimation and the variance of directional distortion are shown in Figure 6 under 500 independent Monte Carlo trials for each value.

2.3.4. Position Distortion. When the vector sensor array is formed in a certain way, the position of its installation location will inevitably produce a deviation, that is, the position error of the array element. At this time, the position error of array element affects the wave path difference and phase difference, so it influences orientation estimation of array.

For uniform linear array, the position error of the $m$ th sensor is $\left(\Delta x_{m}, \Delta y_{m}\right)$. The wave path difference between the $m$ th and the referenced sensor is $\left((m-1) d+\Delta x_{m}\right) \cos \theta+$ $\Delta y_{m} \sin \theta$, where $d$ represents the interelement spacing. At this point, the corresponding phase difference is $-2 \pi$ $\left[\left((m-1) d+\Delta x_{m}\right) \cos \theta+\Delta y_{m} \sin \theta\right] / \lambda$. Compared with no error, the phase error of the $m$ th sensor is $-2 \pi\left[\left(\Delta x_{m} \cos \theta+\right.\right.$ $\left.\Delta y_{m} \sin \theta\right) / \lambda$.

Therefore, the error of the array element will eventually lead to the phase error of the array element. For the error term, the vector sensor is a whole. So the phase error of the acoustic pressure and velocity of the same

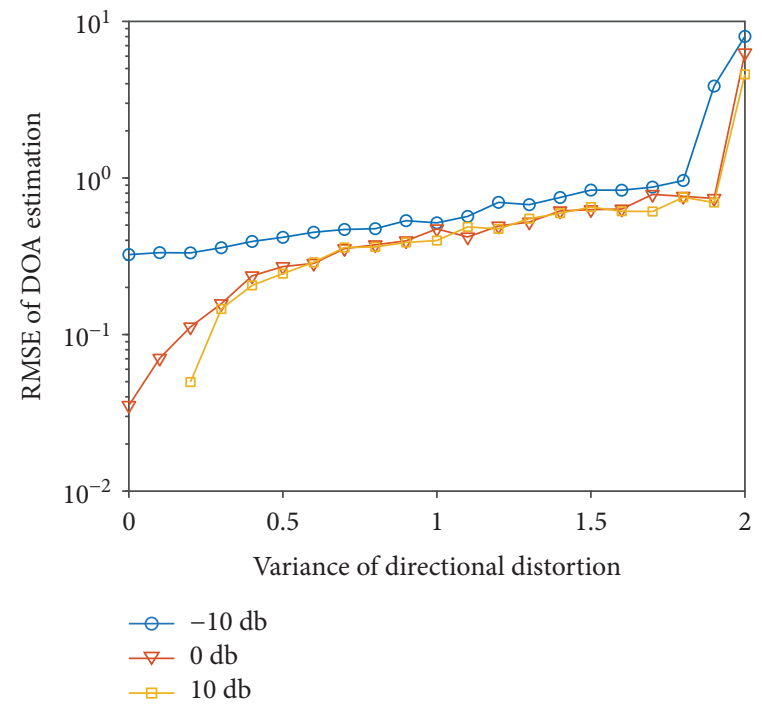

FIgURE 6: The curves between RMSE of DOA estimation and the variance of directional distortion with different SNRs.

sensor are consistent. At this point, the effect of the whole array is

$$
\Psi=\operatorname{diag}\left(\varphi_{1}, \varphi_{1}, \varphi_{1}, \varphi_{2}, \varphi_{2}, \varphi_{2}, \cdots, \varphi_{M}, \varphi_{M}, \varphi_{M}\right),
$$

where $\varphi_{m}=e^{-j 2 \pi\left(\Delta x_{m} \cos \theta+\Delta y_{m} \sin \theta\right)} / \lambda, m=1,2, \cdots, M$.

Similar to the above simulation conditions, the curves between the RMSE of DOA estimation and the variance of directional distortion are shown in Figure 7 under 500 independent Monte Carlo trials for each value. It can be seen that the position distortion has the greatest influence on the performance of DOA estimation.

2.3.5. Comprehensive Distortion. Considering the effects of the above four kinds of errors, the output of the $m$ th sensor is

$\mathbf{q}_{m}=\left[\begin{array}{c}q_{m p} \\ q_{m v_{x}} \\ q_{m v_{y}}\end{array}\right]=\left[\begin{array}{c}g_{m p} e^{j \phi n p} \varphi_{m} \\ g_{m v_{x}} e^{j \phi n v_{x}} \varphi_{m} \cos \left(\theta+\Delta \theta_{m x}\right) \\ g_{m v_{y}} e^{j \phi n p} \varphi_{m} \sin \left(\theta+\Delta \theta_{m x}+\Delta \theta_{m y}\right)\end{array}\right], m=1,2, \cdots, M$.

Then, the output of the whole array is

$$
\mathbf{Z}(t)=\Gamma \mathbf{A}(\theta) \mathbf{S}(t)+\mathbf{n}(t)
$$

where $\mathbf{\Gamma}=\mathbf{G} \cdot \boldsymbol{\Phi} \cdot \mathbf{Q} \cdot \boldsymbol{\Psi}$ represents the comprehensive distortional matrix of array.

Suppose an uniform line array is composed of 6 vector sensors, interelement spacing is half wavelength, and the adding noise is Gaussian white noise and assumes SNR being $0 \mathrm{~dB}$ and the number of snapshots being 500 .

Suppose two signals from $30^{\circ}$ and $100^{\circ}$ incident on the array, the SNR is $10 \mathrm{~dB}$, the spectrum of MUSIC algorithm with no errors, and the comprehensive error shown in Figure 8 , it can be seen that the MUSIC algorithm almost 


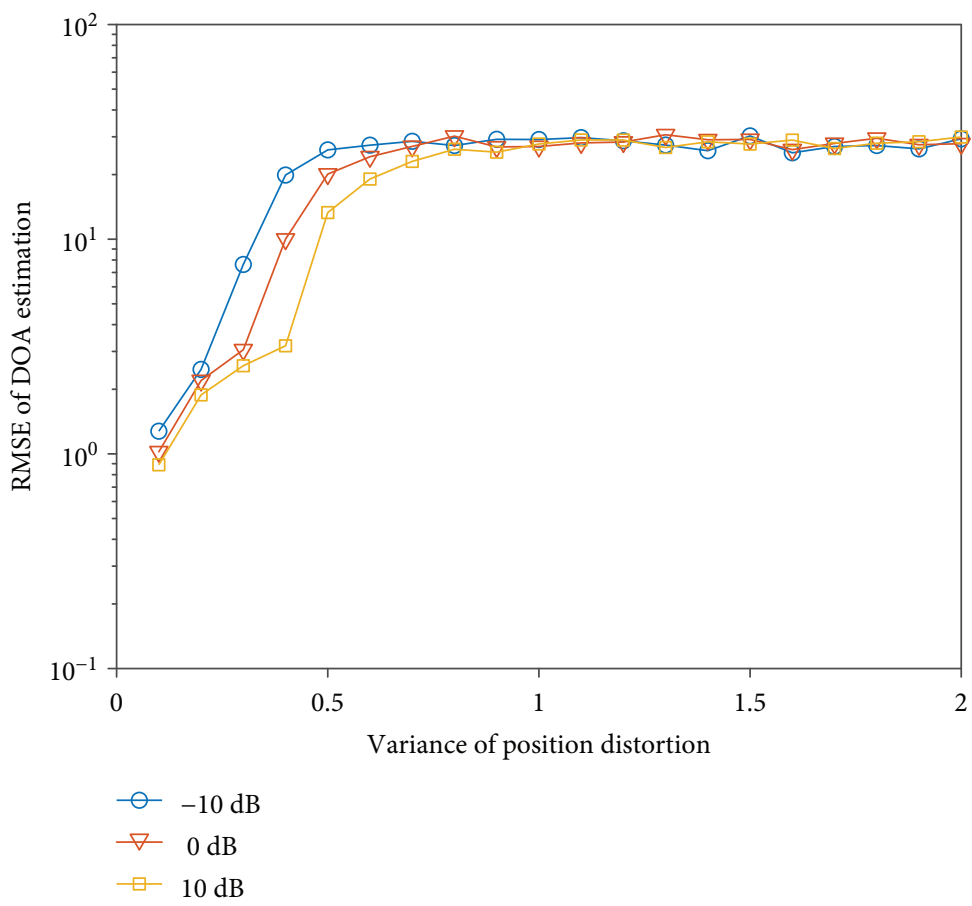

FIGURE 7: The curves between RMSE of DOA estimation and the variance of position distortion with different SNRs.

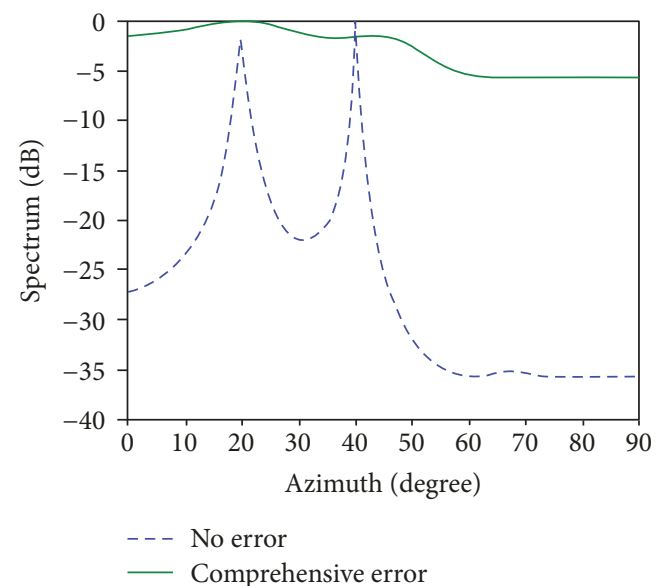

FIGURE 8: The MUSIC spectrum in the influence of comprehensive error.

failed to determine the orientation of the signal under the comprehensive effects of various errors.

\section{Error Self-Calibration Algorithm for Vector Sensor Array}

The statistical analysis and simulation results show that all errors can be attributed to the amplitude and phase distortions of the vector array. The presence of these model errors will affect the DOA estimation of the vector array. The amplitude distortions will change the eigenvalue of the covariance matrix received by the array and then change the peak height and increase the width of the main peak and the height of the side lobe in the spectrum, while the phase error changes the eigenvector of the covariance matrix, which results in the offset of the maximum of the spectrum and the estimation error. On the basis of the classical selfcalibration algorithm for array errors of acoustic pressure $[18,19]$, a self-calibration algorithm for vector sensor array error is proposed.

The basic principle of the self-calibration algorithm is the use of noise subspace and signal subspace orthogonal, even space of noise subspace and array steering vector are orthogonal. Then, the cost function can be obtained by

$$
J=\sum_{i=1}^{N}\left\|\mathbf{U}_{n}^{H} \boldsymbol{\Gamma a}\left(\boldsymbol{\theta}_{i}\right)\right\|^{2}=\sum_{i=1}^{N} \mathbf{a}^{H}\left(\boldsymbol{\theta}_{i}\right) \boldsymbol{\Gamma}^{H} \mathbf{U}_{n} \mathbf{U}_{n}^{H} \boldsymbol{\Gamma} \mathbf{a}\left(\boldsymbol{\theta}_{i}\right) .
$$

The self-calibration of array error can be achieved by the iterative solution for optimizing the cost function $J$, i.e.,

$$
\{\widehat{\Gamma}, \widehat{\theta}\}=\arg \min _{\Gamma, \theta} J
$$

Let

$$
\begin{aligned}
\boldsymbol{\alpha}(\boldsymbol{\theta}) & =\left[\begin{array}{llll}
\operatorname{diag}\left[\mathbf{a}\left(\theta_{1}\right)\right] & & & \\
& \operatorname{diag}\left[\mathbf{a}\left(\theta_{2}\right)\right] & & \\
& & \ddots & \\
& & \operatorname{diag}\left[\mathbf{a}\left(\theta_{N}\right)\right]
\end{array}\right]_{(3 M)^{2} \times(3 M)^{2}}, \\
\boldsymbol{\delta} & =[\operatorname{vec}(\boldsymbol{\Gamma})]_{(3 M)^{2} \times 1},
\end{aligned}
$$


Error self-calibration algorithm for AVS array.

(a) Initialization: $k=0, \widehat{\boldsymbol{\Gamma}}^{(k)}=\boldsymbol{\Gamma}_{0}$

(b) Use the matrix of vector sensor array of amplitude and phase distortion $\widehat{\Gamma}^{(k)}$ and calculate spectrum of MUSIC algorithm based on formula (10)

$P\left(\boldsymbol{\theta} \mid \widehat{\boldsymbol{\Gamma}}^{(k)}\right)=\frac{1}{\mathbf{a}^{H}\left(\theta_{i}\right) \boldsymbol{\Gamma}^{H} \mathbf{U}_{n} \mathbf{U}_{n}^{H} \boldsymbol{\Gamma} \mathbf{a}\left(\theta_{i}\right)}$,

and the azimuth corresponding to the $N$ spectral peaks of the spatial spectrum is used as the DOA estimation $\widehat{\boldsymbol{\theta}}^{(k)}$ of the signal source (c) Fix azimuth estimation $\widehat{\boldsymbol{\theta}}^{(k)}$ and calculate $\widehat{\boldsymbol{\Gamma}}^{(k+1)}$ from the vector $\widehat{\boldsymbol{\delta}}$ by formula (28) $\widehat{\boldsymbol{\Gamma}}^{(k+1)}=\operatorname{diag}\left(\widehat{\boldsymbol{\delta}}^{(k+1)}\right)$

(d) Compute the cost function value by formula (25) to determine whether the convergence will stop with threshold $\varepsilon$, and the convergence condition is

$J^{(k+1)}-J^{(k)} \leq \varepsilon$,

if it is convergent, then the iteration stops. Otherwise, $k=k+1$, go back to (b).

Algorithm 1

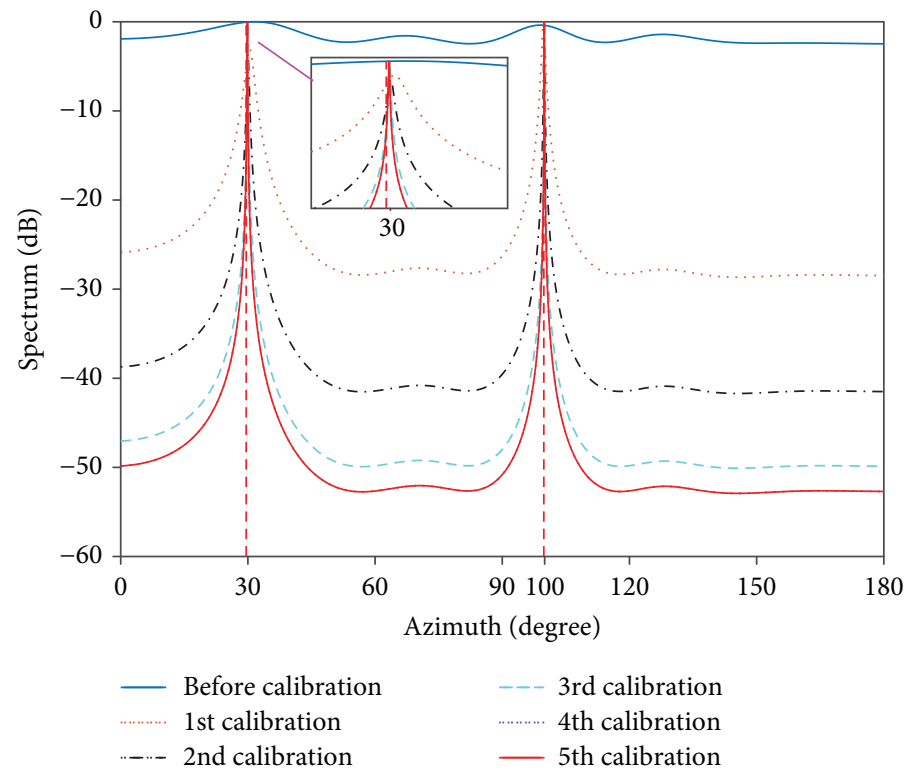

FIgURE 9: The MUSIC spectrum of self-calibration iteration.

TABLE 1: Self-calibration iterative process of array error of vector sensor.

\begin{tabular}{lccc}
\hline Iteration number & Signal 1 & Signal 2 & Cost function value of $J$ \\
\hline 1 & 31.7 & 99 & 10 \\
2 & 30.4 & 99.7 & 0.1703 \\
3 & 30.0 & 99.9 & 0.013 \\
4 & 29.9 & 99.9 & 0.0005 \\
5 & 29.9 & 99.9 & 0.0002 \\
6 & 29.9 & 99.9 & 0.0002 \\
\hline
\end{tabular}

which represent the straightening operation of matrix $\Gamma$, then

$$
\Gamma \mathbf{a}(\theta)=\alpha(\theta) \delta
$$

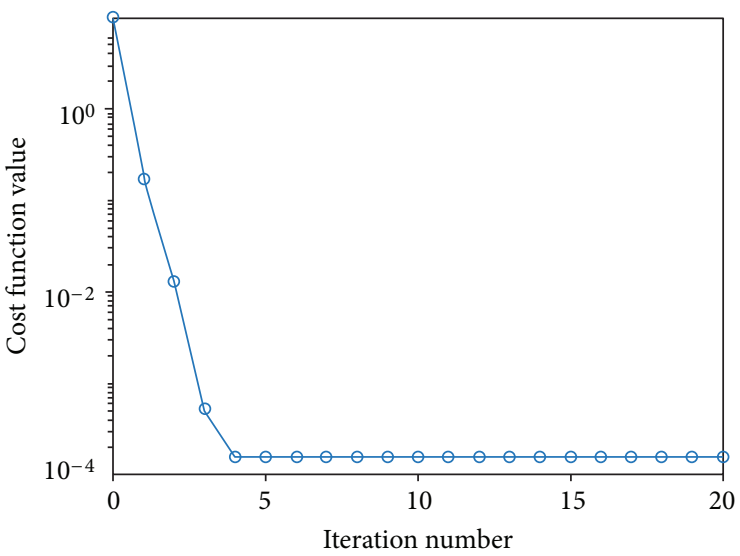

Figure 10: The iteration curve of cost function. 
TABLE 2: Error self-calibration result of vector sensor array.

\begin{tabular}{|c|c|c|c|c|c|c|c|}
\hline & Vector & $\begin{array}{c}\text { Actual amplitude } \\
\text { error }\end{array}$ & $\begin{array}{c}\text { Calibration amplitude } \\
\text { error }\end{array}$ & $\begin{array}{c}\text { Relative error } \\
(\%)\end{array}$ & $\begin{array}{l}\text { Actual phase } \\
\text { error (rad) }\end{array}$ & $\begin{array}{l}\text { Calibration phase } \\
\text { error (rad) }\end{array}$ & $\begin{array}{c}\text { Relative error } \\
(\%)\end{array}$ \\
\hline \multirow{3}{*}{1} & Pressure & 1 & 1 & 0.00 & 0 & 0 & 0.00 \\
\hline & Velocity $x$ & 0.6603 & 0.6514 & 1.35 & -1.3566 & -1.3586 & 0.15 \\
\hline & Velocity $y$ & 1.4775 & 1.487 & 0.64 & 0.7286 & 0.7283 & 0.04 \\
\hline \multirow{3}{*}{2} & Pressure & 0.9364 & 0.9324 & 0.24 & 0.6072 & 0.6315 & 4.00 \\
\hline & Velocity $x$ & 1.3247 & 1.3076 & 1.29 & -0.8283 & -0.8023 & 3.14 \\
\hline & Velocity $y$ & 1.2993 & 1.3060 & 0.52 & 2.8876 & 2.9122 & 0.85 \\
\hline \multirow{3}{*}{3} & Pressure & 0.9073 & 0.9031 & 0.07 & 0.9191 & 0.9728 & 5.84 \\
\hline & Velocity $x$ & 0.0096 & 0.0095 & 1.04 & 0.4058 & 0.4379 & 7.91 \\
\hline & Velocity y & 1.1815 & 1.1892 & 0.65 & 1.9162 & 1.9696 & 2.79 \\
\hline \multirow{3}{*}{4} & Pressure & 1.352 & 1.3486 & 0.25 & -2.4557 & -2.3770 & 3.20 \\
\hline & Velocity $x$ & 0.6237 & 0.6157 & 1.28 & -0.0911 & -0.0812 & 10.87 \\
\hline & Velocity $y$ & 0.9915 & 0.9966 & 0.51 & -0.0247 & -0.0223 & 9.72 \\
\hline \multirow{3}{*}{5} & Pressure & 0.7792 & 0.7786 & 0.0800 & 0.1999 & 0.2072 & 3.65 \\
\hline & Velocity $x$ & 1.8507 & 1.8257 & 1.35 & -1.0383 & -0.9320 & 10.24 \\
\hline & Velocity y & 0.9847 & 0.9912 & 0.66 & 0.5294 & 0.5365 & 1.34 \\
\hline \multirow{3}{*}{6} & Pressure & 1.102 & 1.0992 & 0.25 & -2.2171 & -2.0839 & 6.01 \\
\hline & Velocity $x$ & 0.4761 & 0.47 & 1.28 & 0.1074 & 0.1011 & 5.87 \\
\hline & Velocity $y$ & 0.6323 & 0.6355 & 0.51 & 0.4389 & 0.4722 & 7.59 \\
\hline
\end{tabular}

so the cost function of formula (21) can be deformed to

$$
\begin{aligned}
J & =\sum_{i=1}^{N} \mathbf{a}^{H}\left(\theta_{i}\right) \boldsymbol{\Gamma}^{H} \mathbf{U}_{n} \mathbf{U}_{n}^{H} \boldsymbol{\Gamma} \mathbf{a}\left(\theta_{i}\right)=\boldsymbol{\delta}^{H} \boldsymbol{\Omega}(\boldsymbol{\theta}) \boldsymbol{\delta}, \\
\boldsymbol{\Omega}(\boldsymbol{\theta}) & =\sum_{i=1}^{N} \boldsymbol{\alpha}^{H}\left(\boldsymbol{\theta}_{i}\right) \mathbf{U}_{n} \mathbf{U}_{n}^{H} \boldsymbol{\alpha}\left(\boldsymbol{\theta}_{i}\right) .
\end{aligned}
$$

Hence, we want to minimize formula (22) with respect to $\delta$ under the constraint $\boldsymbol{\delta}^{H} \mathbf{w}=1$, in which

$$
\mathbf{w}=\underbrace{[1,0,0, \cdots, 0]}_{3 M}]^{\mathrm{T}} .
$$

The result of this quadratic minimization problem under linear constraints is well known by Lagrange multiplier method and given by

$$
\boldsymbol{\delta}=\frac{\boldsymbol{\Omega}^{-1}(\boldsymbol{\theta}) \mathbf{w}}{\mathbf{w}^{\mathrm{T}} \boldsymbol{\Omega}^{-1}(\boldsymbol{\theta}) \mathbf{w}} .
$$

The solution of the above optimization problem can be solved by iteration of alternating calculation of parameters $\theta$ and $\Gamma$ (Algorithm 1).

In each step of the iterative optimization process and in every direction and every step of frequency iteration estimation, the cost function $J$ will be reduced so that

$$
J^{(0)} \geq J^{(1)} \geq \cdots \geq J^{(k)} \geq 0
$$

so $J^{(k)}$ is a convergent series and can converge to an optimal solution. In a large number of simulation experiments, we find that the initial value of $\boldsymbol{\Gamma}_{0}$ is taken as the $3 M$-order unit matrix $\mathbf{I}_{3 M}$ and the effect is better; this is equivalent to the choice of initial values which are assumed to be error-free.

\section{Results and Discussion}

4.1. Simulation Experiments. Suppose two signal sources from $30^{\circ}$ and $100^{\circ}$ incident on the uniform linear array are composed of 6 AVSs, where the interelement spacing is half wavelength, adding noise is Gaussian white noise, SNR is $10 \mathrm{~dB}$, and the number of snapshots is 500 . When the amplitude response coefficient satisfies the normal distribution in which the mean value is 1 and variance is 0.5 , the phase response satisfies the normal distribution in which the mean value is 0 and variance is 1 , the error calibration effect of the vector sensor array using Algorithm 1 is shown in Figure 9.

In the iterative process, the cost function value and the position of the two signal sources are estimated as shown in Table 1, and the curve of iteration process is shown in Figure 10. With the decrease of the cost function value, the orientation of the two signal sources is more and more closer to the real orientation. For the threshold $\varepsilon=1 E-4$, the cost function value is 0.002 after the fifth calibration, and its value remains unchanged in the next iteration; so the iterative process converges, it also shows that the convergence speed of the proposed algorithm is very fast. The calibration result 


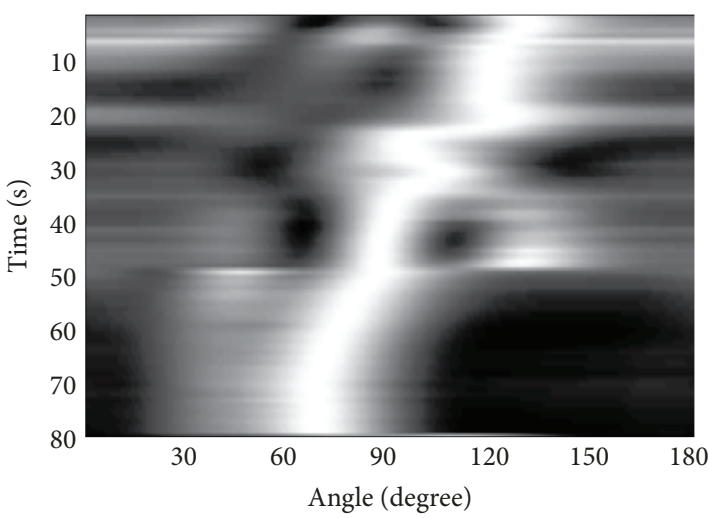

(a) Conventional beamforming

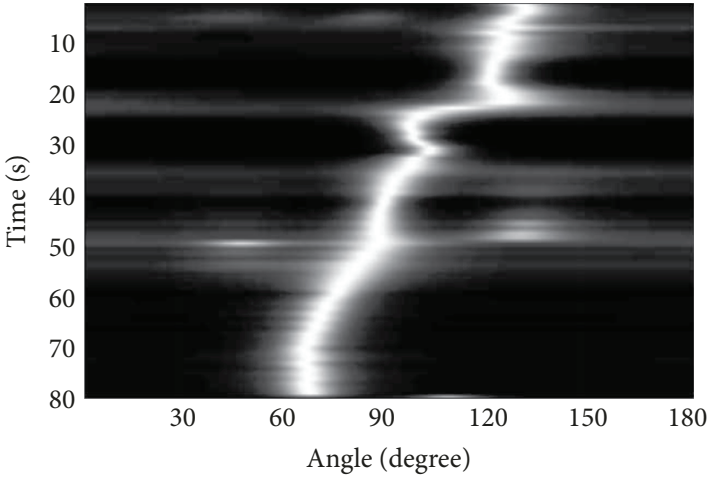

(b) MUSIC algorithm

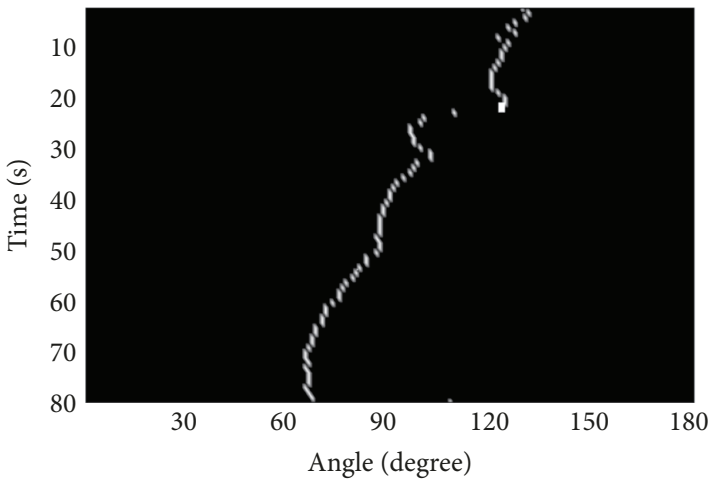

(c) MUSIC algorithm by error self-calibration

FIGURE 11: Displacement process diagrams of three methods.

of the amplitude and phase distortion and relative errors of the sensor array of 6 elements (18 channels) are shown in Table 2. It is seen that the maximum relative error of amplitude error estimate is $1.35 \%$ and the maximum relative error of phase error estimation is $10.87 \%$.

So the results of the algorithm for the calibration of amplitude and phase errors are satisfactory.

4.2. Lake Experiment. The test experiment has been made in Fenhe lake. The line array has been composed of four MEMS vector sensor with interelement spacing being $0.5 \mathrm{~m}$, and it has been fixed underwater $10 \mathrm{~m}$ at the side of the ship. The array's compass could take real-time measurement for its pose to keep the array's horizontality.

The experiment used a motor boat as the moving target, which run from about $130^{\circ}$ to about $70^{\circ}$, tested track time is about $80 \mathrm{~s}$. Broadband noise in which motor boat radiates has been narrowband-filtered as $800 \mathrm{~Hz}$ for the center frequency, once per second. The tracking results of the conventional beamforming, MUSIC algorithm, and MUSIC algorithm by error self-calibration are shown in Figure 11. The results shown that the estimation results of conventional beamforming are very fuzzy; the results of the MUSIC algorithm are clear than the conventional beamforming but not clear in some directions. After using the MUSIC algorithm of the array error self-calibration, it can be very clear to show the operating trajectory of the motor ship.

\section{Conclusion}

In this paper, according to the characteristics of the vector sensor array, the influence of DOA estimation performance by the array's amplitude distortion, phase distortion, array element directional distortion, and position distortion is analyzed, and the vector array signal model under comprehensive error is built. Based on this, an error self-calibration algorithm based on vector array is proposed. Finally, the performance of the algorithm is verified by simulation experiments and lake experiments. These shown that the proposed algorithm has important value for the engineering application of MEMS vector sensor array.

\section{Data Availability}

The data used to support the findings of this study are available from the corresponding author upon request.

\section{Conflicts of Interest}

The authors declare that there is no conflict of interest regarding the publication of this paper.

\section{Acknowledgments}

This research project is supported by the National Nature Science Foundation of China (Grant No. 61127008), the 
Natural Science Foundation of Shanxi Province (Grant Nos. 201701D121012, 201801D121026, and 201701D221121), and the Shanxi Scholarship Council of China (Grant No. 2016088).

\section{References}

[1] A. Nehorai and E. Paldi, "Acoustic vector-sensor array processing," IEEE Transactions on Signal Processing, vol. 42, no. 9, pp. 2481-2491, 1994.

[2] J. He and Z. Liu, "Two-dimensional direction finding of acoustic sources by a vector sensor array using the propagator method," Signal Processing, vol. 88, no. 10, pp. 2492-2499, 2008.

[3] C. Xue, S. Chen, W. Zhang, B. Zhang, G. Zhang, and H. Qiao, "Design, fabrication, and preliminary characterization of a novel mems bionic vector hydrophone," Microelectronics Journal, vol. 38, no. 10-11, pp. 1021-1026, 2007.

[4] C. Xue, Z. Tong, B. Zhang, and W. Zhang, "A novel vector hydrophone based on the piezoresistive effect of resonant tunneling diode," IEEE Sensors Journal, vol. 8, no. 4, pp. 401402, 2008.

[5] G. Zhang, J. Ding, W. Xu et al., "Design and optimization of stress centralized mems vector hydrophone with high sensitivity at low frequency," Mechanical Systems and Signal Processing, vol. 104, pp. 607-618, 2018.

[6] P. Wang, G. J. Zhang, C. Y. Xue, W. D. Zhang, and J. J. Xiong, "Engineering application of mems vector hydrophone and self-adapting root-music algorithm," in 2011 16th International Solid-State Sensors, Actuators and Microsystems Conference, Beijing, China, June 2011.

[7] K. G. Nagananda and G. V. Anand, "Subspace intersection method of high-resolution bearing estimation in shallow ocean using acoustic vector sensors," Signal Processing, vol. 90, no. 1, pp. 105-118, 2010.

[8] K. T. Wong and M. D. Zoltowski, "Self-initiating music-based direction finding in underwater acoustic particle velocity-field beamspace," IEEE Journal of Oceanic Engineering, vol. 25, no. 2, pp. 262-273, 2000.

[9] P. Wang, G. J. Zhang, C. Y. Xue, W. D. Zhang, and J. J. Xiong, "Self-adapting root-music algorithm and its real-valued formulation for acoustic vector sensor array," EURASIP Journal on Advances in Signal Processing, vol. 2012, no. 1, 2012.

[10] E. K. L. Hung, "Matrix-construction calibration method for antenna arrays," IEEE Transactions on Aerospace and Electronic Systems, vol. 36, no. 3, pp. 819-828, 2000.

[11] B. P. Flanagan and K. L. Bell, "Improved array self calibration with large sensor position errors for closely spaced sources," in Proceedings of the 2000 IEEE Sensor Array and Multichannel Signal Processing Workshop. SAM 2000 (Cat. No.00EX410), Cambridge, MA, USA, March 2000.

[12] K. Liu, G. Liang, and G. Zhang, "Exploratory analysis to influence of array errors on vector sensor array beam-forming system," Journal of System Simulation, vol. 24, no. 4, pp. 848-853, 2012.

[13] Y. Liu and B. L. Jin, "A simple method for phase error correction in the vector hydrophone array," Computer Simulation, vol. 27, no. 8, pp. 351-354, 2010.

[14] G. Liang, K. Zhang, and W. Wu, "Correction algorithm of acoustic vector sensor array and DOA estimation based on subspace fitting," Acta Armamentarii, vol. 33, no. 9, pp. 1112-1117, 2012.

[15] H. S. Lim, K. Rameshchandra, and S. T. Wei, "Estimation and compensation of rotation perturbation in linear 2D acoustic vector sensor array," in OCEANS 2011 IEEE Spain, Santander, Spain, June 2011.

[16] G. Liang, K. Zhang, and S. An, "Research on self-calibration algorithm for attitude errors of acoustic vector sensor array," Acta Armamentarii, vol. 35, no. 8, pp. 1236-1242, 2014.

[17] K. Zhang, C. Wang, and F. U. Jin, "Active calibration algorithm for location, gain and phase of acoustic vector sensor array," Journal of Applied Acoustics, vol. 34, no. 5, pp. 457464, 2015.

[18] B. Friedlander and A. J. Weiss, "Direction finding in the presence of mutual coupling," IEEE Transactions on Antennas and Propagation, vol. 39, no. 3, pp. 273-284, 1991.

[19] B. Friedlander and A. J. Weiss, "Effects of model errors on waveform estimation using the music algorithm," IEEE Transactions on Signal Processing, vol. 42, no. 1, pp. 147-155, 1994. 


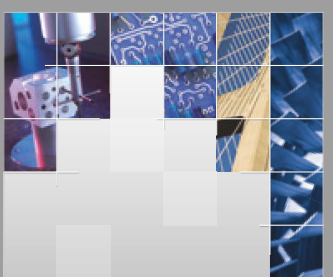

\section{Enfincering}
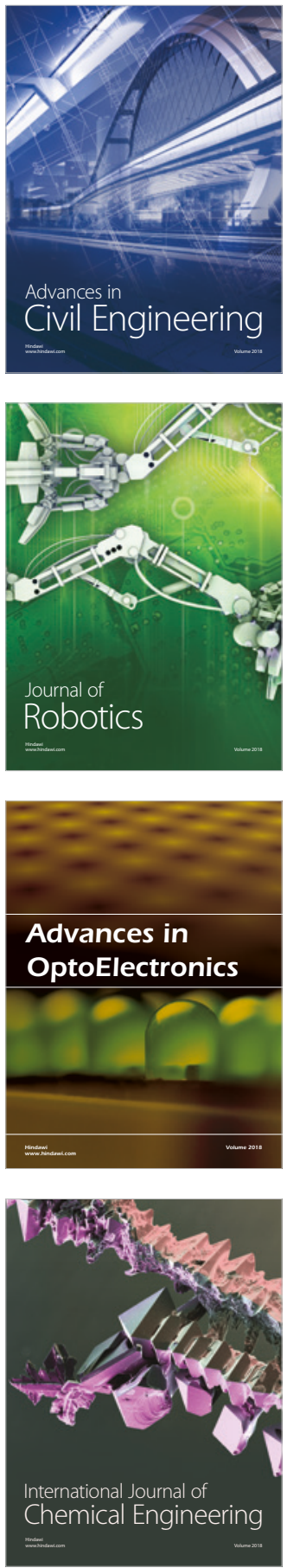

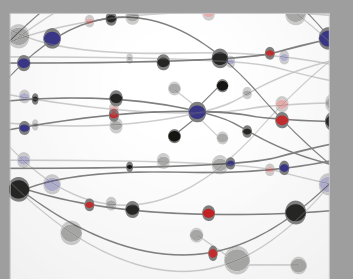

\section{Rotating \\ Machinery}

The Scientific World Journal

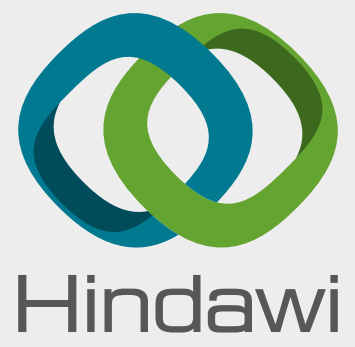

Submit your manuscripts at

www.hindawi.com
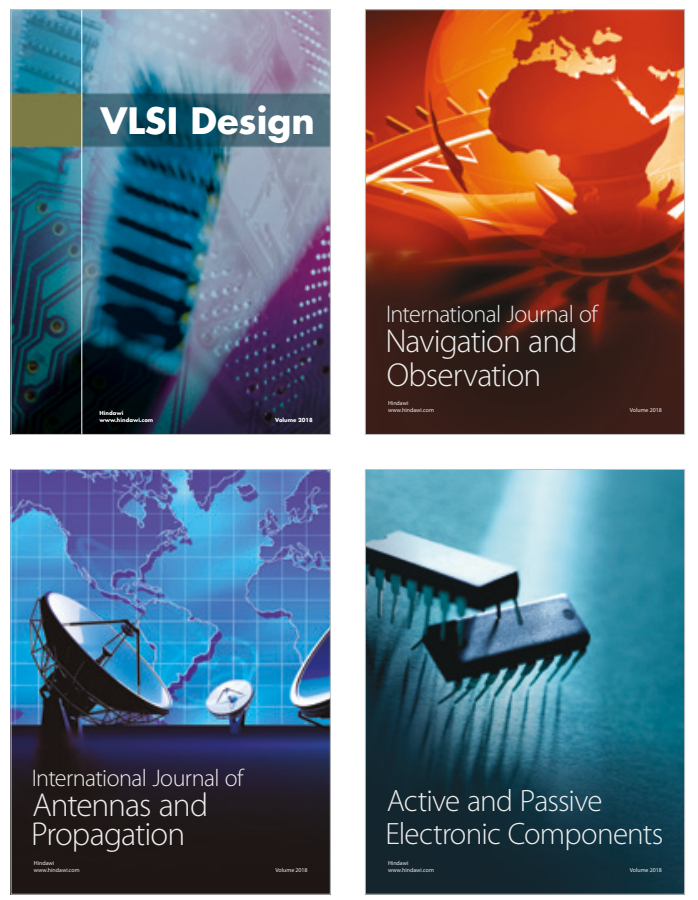
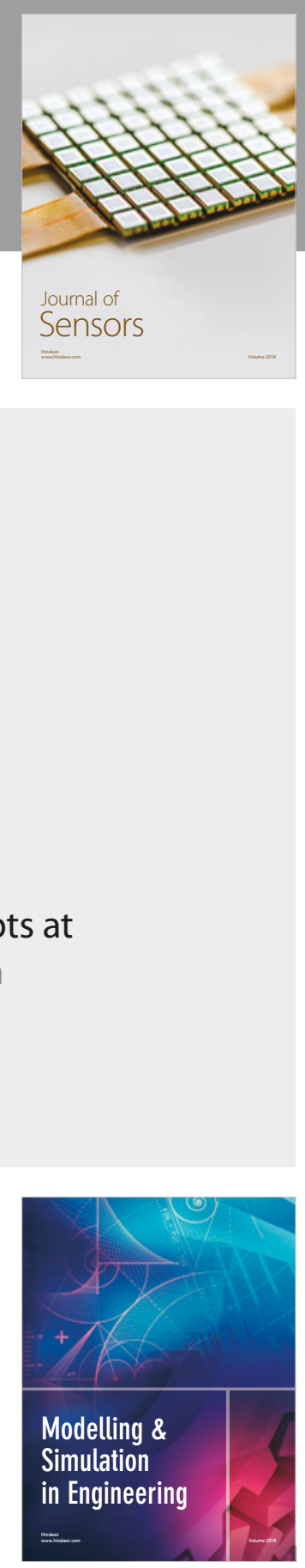

\section{Advances \\ Multimedia}
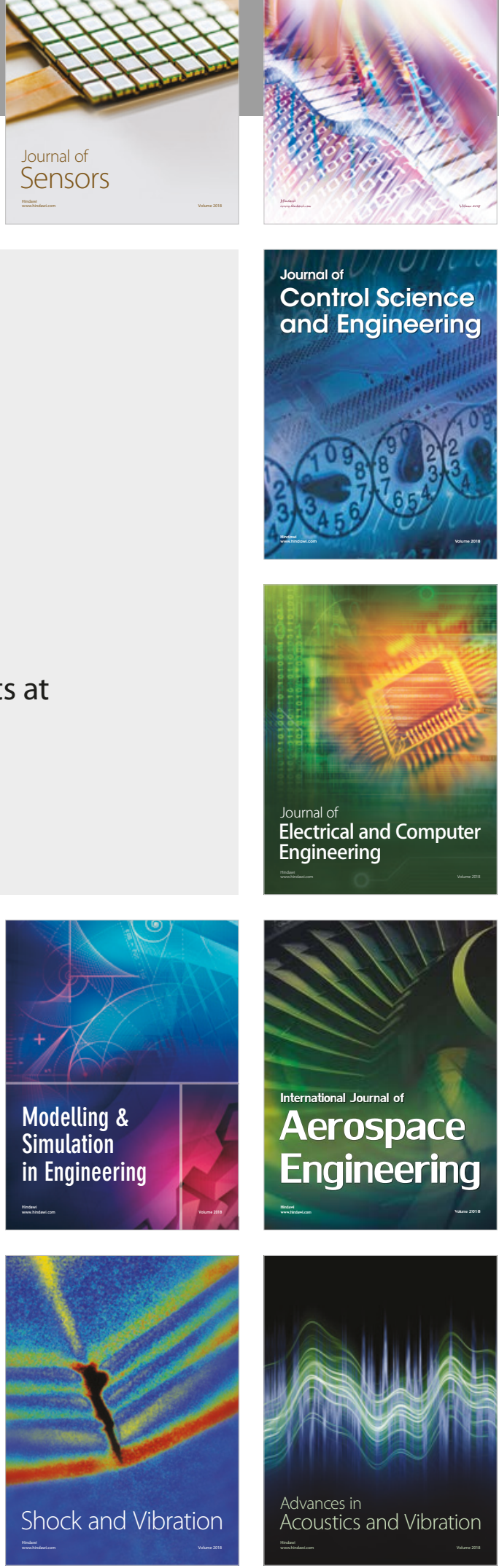